\title{
Critical switching characteristics of three-layered spin valve for different materials and alloys with uniaxial anisotropy
}

\author{
Iuliia Iusipova ${ }^{1,2, *}$ \\ ${ }^{1}$ MIET, General Physics Department, Bld. 1, Shokin Square, Zelenograd, Moscow, Russia, 124498 \\ ${ }^{2}$ IPPM RAS, Department of design of microelectronic components for nanotechnology, 3, Sovetskaya Street, Zelenograd, \\ Moscow, Russia, 124365
}

\begin{abstract}
We analyze the dependence of the current density and magnetic field switching on the magnetic parameters of the material of the ferromagnetic layers of the spin valve. Comparison of critical characteristics of the spin valve with longitudinal anisotropy of ferromagnetic layers fabricared of different materials showed that the promising materials for the fabrication of spin valve are cobalt, iron, their alloys, ferroborates of cobalt and alloys of cobalt with gadolinium. For these materials we produced and analyzed the bifurcation diagrams of equations describing the switching process of the spin valve. Based on the study of the dynamics of the magnetization vector we obtained the numerical evaluation of time switching.
\end{abstract}

\section{Introduction}

Currently, the existing types of memory are approaching to the limits of their possibilities, and intensive development of new, particularly non-volatile types of memory is observed. In 2016, the IBM Corporation, in cooperation with the company Samsung has demonstrated a new magnetoresistive random access memory (MRAM), the diameter of the cells of which is 11 nanometers. The switching time of this cell is superior to all existing types of memory and is only 10 nanoseconds [1]. Magnetoresistive random access memory (MRAM), has many advantages over other types of memory, however, has one significant drawback: the values of current density and magnetic field that must be applied to switch the free layer of the spin valve, which is included in a memory cell of MRAM, are too large

The present work investigates the influence of the magnetic characteristics of the material of the ferromagnetic layers of the spin valve on the dynamics of the magnetization vector of the free layer. The aim of this study is the search of ferromagnetic material, providing better switching characteristics of the spin valve.

\section{The mathematical model of a spin valve}

The transverse section of a spin valve is a square with side $11 \mathrm{~nm}$. The thickness of the free layer $d$ is $2 \mathrm{~nm}$, the thickness of the pinned layer is $5 \mathrm{~nm}$, the thickness of the non-magnetic interlayer is $1.2 \mathrm{~nm}$. Physical basics of spin valve under the action of spin-polarized current was first described in the work of J.C.Slonczewski [2].
The dynamic equation of the magnetization in the free layer of the spin valve in the form of the canonical dynamical system has the form (see e.g. [3-5]):

$$
\frac{\partial \mathbf{m}}{\partial \tau}=-\left[\mathbf{m} \times \mathbf{h}^{e f f}\right]+\alpha \mathbf{h}^{e f f}-\alpha \mathbf{m}\left(\mathbf{m}, \mathbf{h}^{e f f}\right),
$$

where $\mathbf{m}$ is the magnetization of the free layer and the effective field $\mathbf{h}^{\text {eff }}$ is determined by the relation (2).

$$
\mathbf{h}^{\text {eff }}=\left(\begin{array}{c}
h_{x} \\
h_{y} \\
h_{z}
\end{array}\right)=\left(\begin{array}{c}
h+k m_{x} \\
-j G m_{z} \\
j G m_{y}-m_{z}
\end{array}\right) .
$$

We denote in (1), (2) $h=\frac{H}{M_{s}} \quad \tau=\frac{t \gamma \mu_{0} M_{s}}{\left(1+\alpha^{2}\right)}$, $j=\frac{J \hbar}{d e \mu_{0} M_{s}^{2}}, \quad k=\frac{2 K}{\mu_{0} M_{s}^{2}}$, where $H$ is the external magnetic field, $M_{s}$ is the saturation magnetization, $t$ is the time, $\mu_{0}$ is the magnetic constant, $\alpha$ is the coefficient of dissipation, $\gamma$ is the gyromagnetic ratio, $d$ is the thickness of the free layer, $e$ is the elementary charge, $\hbar$ is the Planck constant, $K$ is the coefficient of anysotropy.

The impact of the spin-polarized current on the dynamics of the magnetization in such model is described by a current term in the form of Slonczewski Berger. The coefficient of the current polarization included in the expression for that term is

$$
G=\frac{4 P^{3 / 2}}{(1+P)^{3}(3+\mathbf{m} \cdot \mathbf{s})-16 P^{3 / 2}}=\frac{c}{b+m_{x}},
$$


here $\quad c=\frac{4 P^{3 / 2}}{(1+P)^{3}}, b=3-4 c, \quad P=\frac{n_{\uparrow}-n_{\downarrow}}{n_{\uparrow}+n_{\downarrow}} \quad$ is $\quad$ a parameter of the polarization, $\mathbf{s}$ is the magnetization of the the magnetization of the pinned layer [2]. We consider in this work a case of the longitudinal anysotropy where $\mathbf{s}=(1,0,0)$.

In the coordinate record the system (1) has a view

$$
\begin{aligned}
& \dot{m}_{x}=-j\left(m_{y}^{2}+m_{z}^{2}\right) G+\alpha\left(h+k m_{x}\right)- \\
& -\alpha m_{x} L+m_{y} m_{z}, \\
& \dot{m}_{y}=j m_{x} m_{y} G-m_{x} m_{z}-h m_{z}-k m_{x} m_{x}- \\
& -\alpha j m_{z} G-\alpha m_{y} L, \\
& \dot{m}_{z}=m_{y}\left(h+k m_{x}\right)+\alpha\left(j m_{y} G-m_{z}\right)- \\
& -\alpha m_{z} L+j m_{x} m_{z} G,
\end{aligned}
$$

where $L=h m_{x}+k m_{x}^{2}-m_{z}^{2}, m_{x}, m_{y}, m_{z}$ are projections of the vector of magnetization of the free layer of spin velve on the axis $O X, O Y$ and $O Z$ respectively.

The important characteristics of the dynamics of the magnetization are singular points of system (3), i.e. the equilibrium points of the magnetization of the valve structure. In works [3-5] the following expressions for calculation of the coordinates of singular points were obtained

$$
\begin{aligned}
& m_{x}^{2}-1=0, \\
& m_{x}{ }^{4}\left(k^{2}+k\right)+m_{x}^{3}\left(h+2 b k^{2}+2 h k+2 b k\right)+ \\
& +m_{x}{ }^{2}\left(h^{2}+4 h b k+2 h b+b^{2} k^{2}+b^{2} k+c^{2} j^{2}\right)+ \\
& +m_{x}\left(h b^{2}+2 h^{2} b+2 h b^{2} k\right)+h^{2} b^{2}=0 .
\end{aligned}
$$

Equation (4A) corresponds to two singular points that are in the system for all currents and fields, equation (4B) allows to calculate the coordinates of other singular points, depending on the current and field and to determine areas of their existence. In our cosideration, we were interested in the existence in the system (3) trajectory connecting the equilibrium points $T_{1,2}( \pm 1,0,0)$ - in other words, the switching mode. Such a trajectory can exist only if the instability of the initial position of the magnetization vector corresponding to the point $T_{1}(+1,0,0)$, and stability of the final position of the magnetization vector - point $T_{2}(-1,0,0)$.

In works [3-5] expressions for determining the stability of the point $T_{1}(+1,0,0)$ were obtained :

$$
\begin{aligned}
& \frac{\alpha d e M_{S} \mu_{0}}{\hbar G} H-J+\frac{\alpha d e}{\hbar G}\left(\frac{4 K+M_{S}^{2} \mu_{0}^{2}}{2 \mu_{0}}\right)=0 \\
& \left(\frac{H+\frac{4 K+M_{S}^{2} \mu_{0}}{2 M_{S}}}{\frac{M_{S}}{2}}\right)^{2}+\left(\frac{J}{\frac{d e M_{S}^{2} \mu_{0}}{2 G \hbar}}\right)^{2}-1=0
\end{aligned}
$$

Expressions (5a) and (5b) are responsible for the minimum switching current of the spin valve. Expression (5a) is the canonical form of the equation of the straight line separating the regions in which the point $T_{1}(+1,0,0)$ is a stable or an unstable focus, and which intersects the axis at the point

$$
J_{\min }=\frac{\alpha d e}{\hbar G} c, \text { где } c=\left(\frac{4 K+M_{S}^{2} \mu_{0}^{2}}{2 \mu_{0}}\right) .
$$

The expression $(5 b)$ is the canonical form of the equation of an ellipse within which the point $T_{1}(+1,0,0)$ has the form of a saddle, and which crosses the $H$-axis at the points with coordinates $H_{\min }=\frac{-2 K}{M_{s} \mu_{0}} \quad$ and $H_{\max }=-\frac{2 K+M_{s}^{2} \mu_{0}}{M_{s} \mu_{0}}$. Details of the bifurcation analysis was presented in works [3-5].

The decrease of the switching current can be done in several ways :

1) applying a magnetic field directed opposite to the magnetization of the pinned layer;

2) reducing the thickness of the free layer;

3 ) choosing the manufacturing material of the free layer of the spin valve, the magnetic properties of which require minimal stability region of point $T_{1}(+1,0,0)$.

\section{The influence of the magnetic properties of ferromagnetic materials on threshold fields and on switching currents of spin valve}

Consider an influence of the magnetic properties of materials constituting the ferromagnetic layers of the spin valve, on the threshold switching current for zero magnetic field $J_{\min }$ and minimum magnetic switching field $H_{\min }$ for zero current. For this we have chosen the following materials:

1) ferroborates of cobalt (they possess the best magnetic properties for reducing the switching current due to the high value of the polarization parameter): $\mathrm{Fe}_{60} \mathrm{Co}_{20} \mathrm{~B}_{20}$ which was annealed at the temperature $450^{\circ} \mathrm{C}$, and $\mathrm{Fe}_{40} \mathrm{Co}_{40} \mathrm{~B}_{20}$ and $\mathrm{Fe}_{70} \mathrm{Co}_{30}$ which were annealed at the temperature $300^{\circ} \mathrm{C}$;

2) alloys of cobalt with gadolinium (they possess the best magnetic properties for reducing the switching magnetic field: $\mathrm{Co}_{80} \mathrm{Gd}_{20}$ and $\mathrm{Co}_{93} \mathrm{Gd}_{7}$ which were annealed at the temperature $200^{\circ} \mathrm{C}$;

3) materials, production of mono-crystal membrans of which has the lowest complexity and is less expensive: cobalt, iron.

Magnetic properties of the considered materials are given in Table 1. 
Table 1. The saturation magnetization $M_{s} \mu_{0}$, the coefficient of anisotropy $K$, the spin polarization $P$ and the coefficient of dissipation $\alpha$ for different materials.

\begin{tabular}{|l|c|c|c|c|}
\hline Material $\left.{ }^{*}\right)$ & $\begin{array}{c}M_{s} \mu_{0}, \\
\mathrm{~T}\end{array}$ & $K, \frac{\mathrm{J}}{\mathrm{m}^{3}}$ & $P$ & $\alpha$ \\
\hline $\begin{array}{l}\text { Co (cobalt) } \\
{[6]}\end{array}$ & 1,76 & $5,3 \cdot 10^{5}$ & 0,35 & 0,020 \\
\hline $\begin{array}{l}\mathrm{Fe} \text { (iron) } \\
{[6,7]}\end{array}$ & 2,15 & $4,8 \cdot 10^{4}$ & 0,40 & 0,008 \\
\hline $\begin{array}{l}\mathrm{Fe}_{70} \mathrm{Co}_{30} \\
{[7-9]}\end{array}$ & 2,40 & $3,5 \cdot 10^{4}$ & 0,55 & 0,015 \\
\hline $\begin{array}{l}\mathrm{Fe}_{60} \mathrm{Co}_{20} \mathrm{~B}_{20} \\
{[10-12]}\end{array}$ & 1,96 & $2,1 \cdot 10^{5}$ & 0,53 & 0,040 \\
\hline $\begin{array}{l}\mathrm{Fe}_{40} \mathrm{Co}_{40} \mathrm{~B}_{20} \\
{[13,14]}\end{array}$ & 1,30 & $3,4 \cdot 10^{-2}$ & 0,52 & 0,010 \\
\hline $\begin{array}{l}\mathrm{Co}_{93} \mathrm{Gd}_{7} \\
{[15]}\end{array}$ & 1,21 & $1,88 \cdot 10^{3}$ & 0,30 & 0,020 \\
\hline $\begin{array}{l}\mathrm{Co}_{80} \mathrm{Gd}_{20} \\
{[15]}\end{array}$ & 0,10 & $1,38 \cdot 10^{3}$ & 0,10 & 0,020 \\
\hline
\end{tabular}

*) In square brackets references to the data sources are given

Figure 1 shows the line of stability of the point $T_{1}(+1,0,0)$ for various materials. Switching of spin valve is possible only in the region above the line whose equation is has the form (5a), and within semi-ellipse determined by formula (5b). Table 2 shows the values of the minimum switching currents for zero magnetic field $J_{\min }$ and minimum magnetic field for zero current $H_{\min }$ for various materials. Note that, from physical considerations, there is a number of restrictions on the limiting value of the density of the injection current $J$ and an external magnetic field $H$, associated with electromigration and heating of the sample, which we did not consider in our calculation.

As can be seen from Figure 1, the line of stability of $\mathrm{Co}_{80} \mathrm{Gd}_{20}$ has the minimum slope, in this case, accordingly, there is a minimum threshold current $J_{\min }$ at zero magnetic field (see Table 2). The memory cells MRAM produced by technology with $10 \mathrm{~nm}$ by IBM in partnership with Samsung, use current of density $J=7,89 \cdot 10^{10} \frac{\mathrm{A}}{\mathrm{m}^{2}} \quad[1]$ in the switching, which is significantly more than the estimated values $J_{\min }$ for $\mathrm{Co}_{80} \mathrm{Gd}_{20}$.

Based on figure 1 and table 2 we can conclude that the smallest value of the magnetic field switching at zero current has alloy $\mathrm{Fe}_{40} \mathrm{Co}_{40} \mathrm{~B}_{20}$.

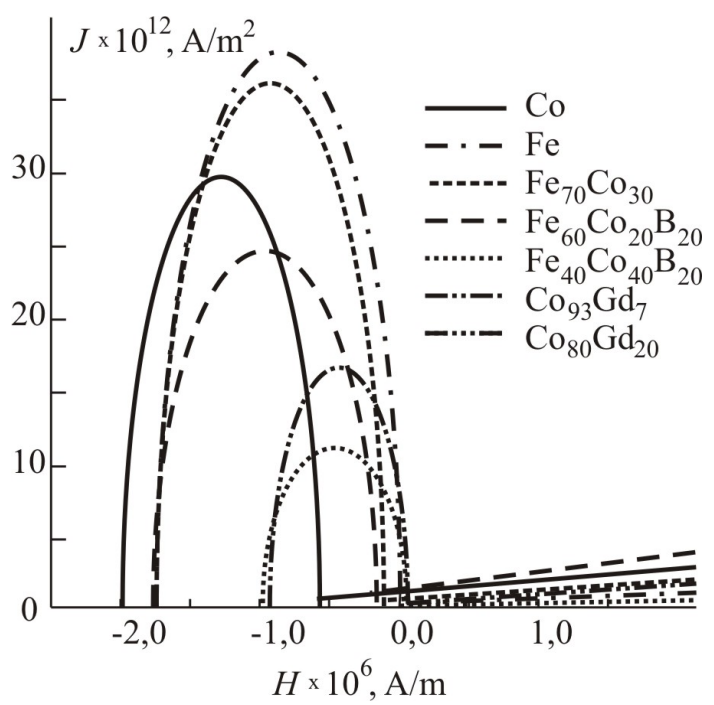

a

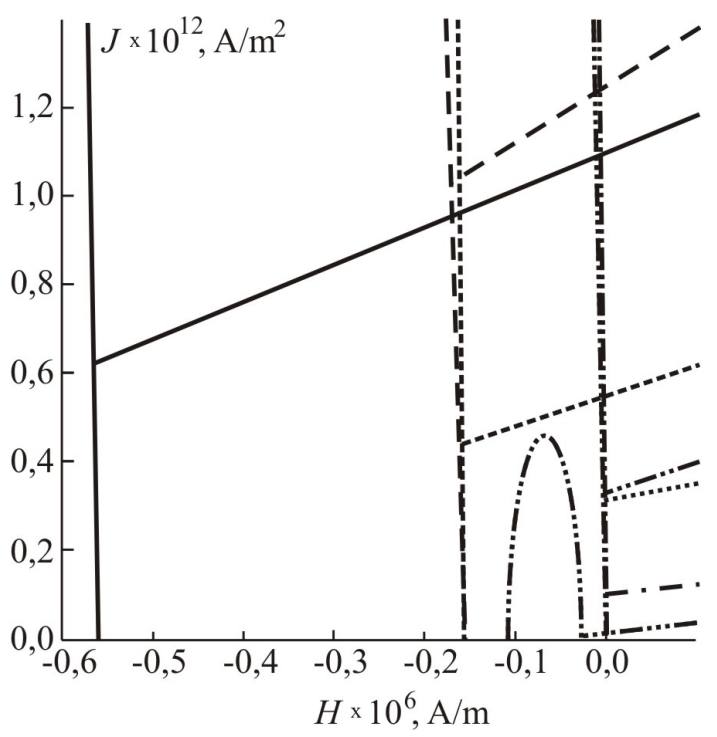

b

Fig. 1. The stability line of singular point $T_{1}(+1,0,0)$ for cobalt (solid line), iron (dash-dotted line), $\mathrm{Fe}_{70} \mathrm{Co}_{30}$ (dashed line with short strokes), $\mathrm{Fe}_{60} \mathrm{Co}_{20} \mathrm{~B}_{20}$ (the dashed line with long dashes), $\mathrm{Fe}_{40} \mathrm{Co}_{40} \mathrm{~B}_{20}$ (dotted line), $\mathrm{Co}_{93} \mathrm{Gd}_{7}$ (dashdotted line with two dots), $\mathrm{Co}_{80} \mathrm{Gd}_{20}$ (dash-dotted line with three dots); a -in the range $H[-2,5 ; 2] \times 10^{6} \frac{\mathrm{A}}{\mathrm{m}}$ and in the current range $J[0 ; 40] \times 10^{12} \frac{\mathrm{A}}{\mathrm{m}^{2}} ; \mathrm{b}-$ in the range $H[-0,6 ; 0,1] \times 10^{6} \frac{\mathrm{A}}{\mathrm{m}}$ and in the current range $J[0 ; 1,4] \times 10^{12} \frac{\mathrm{A}}{\mathrm{m}^{2}}$ 
Table 2. Threshold currents $J_{\min }$ and magnetic fields $H_{\min }$ of switching of the spin valve for various materials

\begin{tabular}{|l|c|c|}
\hline \multicolumn{1}{|c|}{ Material } & $J_{\min }, \frac{\mathrm{A}}{\mathrm{m}^{2}}$ & $H_{\min }, \frac{\mathrm{A}}{\mathrm{m}}$ \\
\hline $\mathrm{Co}$ (cobalt) & $1,12 \cdot 10^{12}$ & $-6,02 \cdot 10^{5}$ \\
\hline $\mathrm{Fe}$ (iron) & $3,38 \cdot 10^{11}$ & $-4,47 \cdot 10^{4}$ \\
\hline $\mathrm{Fe}_{70} \mathrm{Co}_{30}$ & $5,79 \cdot 10^{11}$ & $-2,92 \cdot 10^{4}$ \\
\hline $\mathrm{Fe}_{60} \mathrm{Co}_{20} \mathrm{~B}_{20}$ & $1,31 \cdot 10^{12}$ & $-2,14 \cdot 10^{5}$ \\
\hline $\mathrm{Fe}_{40} \mathrm{Co}_{40} \mathrm{~B}_{20}$ & $1,15 \cdot 10^{11}$ & $-5,17 \cdot 10^{-1}$ \\
\hline $\mathrm{Co}_{93} \mathrm{Gd}_{7}$ & $3,89 \cdot 10^{11}$ & $-3,13 \cdot 10^{3}$ \\
\hline $\mathrm{Co}_{80} \mathrm{Gd}_{20}$ & $2,66 \cdot 10^{9}$ & $-2,76 \cdot 10^{4}$ \\
\hline
\end{tabular}

\section{The calculation of the switching time}

To estimate the time of switching of the MRAM cell, the dynamics of the process of switching the spin valve from parallel to antiparallel state was simulated. Table 3 presents the values of the time of switching of the spin valve which is included in the MRAM memory cell and contains the spin valve of different materials.

Table 3. The switching time of spin valve (in nano-seconds) for different materials

\begin{tabular}{|c|c|c|}
\hline Material & $H=0$ & $J=0$ \\
\hline Co (cobalt) & 187,2 & 14,5 \\
\hline $\mathrm{Fe}$ (iron) & 339,2 & 91,9 \\
\hline $\mathrm{Fe}_{70} \mathrm{Co}_{30}$ & 171,5 & 21,1 \\
\hline $\mathrm{Fe}_{60} \mathrm{Co}_{20} \mathrm{~B}_{20}$ & 52,6 & 79,4 \\
\hline $\mathrm{Fe}_{40} \mathrm{Co}_{40} \mathrm{~B}_{20}$ & 564,0 & 4690,9 \\
\hline $\mathrm{Co}_{93} \mathrm{Gd}_{7}$ & 79,38 & 63,85 \\
\hline $\mathrm{Co}_{80} \mathrm{Gd}_{20}$ & 960,6 & 105,5 \\
\hline
\end{tabular}

As Table 3 shows, the lowest value of the switching time corresponds to the spin valve of cobalt at zero current. However, switching modes, using a magnetic field, suggest the complication of the production of the chip of magnetoresistive memory MRAM, since the application of the magnetic field requires further massive tire. Therefore, the priority mode of switching by the electric current (which gives the most high-speed) is a mode for the spin valve based on $\mathrm{Fe}_{60} \mathrm{Co}_{20} \mathrm{~B}_{20}$.

\section{Conclusion}

Thus, in the present work, we conducted an analysis of the dependence of the current switching on characteristics of the materials of magnetic layers of the spin valve. Based on this analysis it was found that the promising materials for magnetic layers are cobalt, iron, and compounds such as $\mathrm{Fe}_{60} \mathrm{Co}_{20} \mathrm{~B}_{20}$ (which were annealed at the temperature $450^{\circ} \mathrm{C}$ ), $\mathrm{Fe}_{40} \mathrm{Co}_{40} \mathrm{~B}_{20}$ and $\mathrm{Fe}_{70} \mathrm{Co}_{30}$ (which were annealed at the temperature $300^{\circ} \mathrm{C}$ ); $\mathrm{Co}_{80} \mathrm{Gd}_{20}$ and $\mathrm{Co}_{93} \mathrm{Gd}_{7}$ (which were annealed at the temperature $200^{\circ} \mathrm{C}$ ). We investigated the dynamics of the magnetization vector of the free layer of the spin valve fabricated on the basis of these materials. As a result of the calculation of the critical currents and fields, as well as the switching time for each mode without accounting physical restrictions on the values of current density and magnetic field, it is concluded that the most suitable for the manufacture of spin valve contained in the memory cell of MRAM, the materials can serve two alloys, namely $\mathrm{Fe}_{60} \mathrm{Co}_{20} \mathrm{~B}_{20}$ and $\mathrm{Co}_{80} \mathrm{Gd}_{20}$ which were annealed at the temperatures $300^{\circ} \mathrm{C}$ and $200^{\circ} \mathrm{C}$ respectively.

\section{References}

1. J.J. Nowak, R.P. Robertazzi, J.Z. Sun et. all., IEEE Magnetics Letters, 7, 3102604 (2016)

2. J.C. Slonczewski, Journal of Magnetism and Magnetic Materials, 159, L1 (1996)

3. N.V. Ostrovskaya, V.A. Skidanov, Iu.A. Iusipova, Solid State Phenomena, 233-234, 431 (2015)

4. N.V. Ostrovskaya, V.A. Skidanov, Iu.A. Iusipova, Proceedings of IPPM RAS Ed. Stempkovskiy A.L., Moscow: IPPM RAS, 199 (2016)

5. N.V. Ostrovskaya, V.A. Skidanov, Iu.A. Iusipova, Computer Research and Modeling, 8, 4, 605 (2016)

6. R. Skomski, Simple Models of Magnetism (Oxford university press, New York, 2008)

7. T. Kawai1, Y. Asai1, M. Ohtake et. all., EPJ Web of Conferences, 40, 13001 (2013)

8. J.S. Moodera, C. Tanaka et. all., Philosophical Magazine B, 80, 2, 195 (2000)

9. A.P. Babichev, N.A. Babushkina, et. al, Physical quantities: Reference book Ed. Grigor'ev I.S., Meylihov E.Z. (Energoatomizdat, Moscow, 1991)

10. S.X. Huang, T.Y. Chen, C.L. Chien, Appl. Phys. Lett., 92, 242509 (2008)

11. M.H. Nguyen, C.F. Pai, K.X. Nguyen et. all., Appl. Phys. Lett., 106, 222402 (2015)

12. S. Ikeda, K. Miura, H. Yamamoto et. all., Nature Materials, 9, 721 (2010)

13. J. Hayakava, S. Ikeda, Y.M. Lee, et. all., Jap. J. Appl. Phys., 44(2), 37-41, L1267 (2005)

14. H. Almasi, D.R. Hickey, T.Newhouse-Illige et. all., Appl. Phys. Lett., 106, 182406 (2015)

15. C. Kaiser, S.S. PapWorth Parkin United States Patent № US007230265B2 (2007) 